\title{
Target arm affinities determine preclinical efficacy and safety of anti-HER2/CD3 bispecific antibody
}

\author{
Karin Staflin, Christina L. Zuch de Zafra, Leah K. Schutt, Vanessa Clark, Fiona Zhong, \\ Maria Hristopoulos, Robyn Clark, Ji Li, Mary Mathieu, Xiaocheng Chen, Jennifer Johnston, \\ Justin Low, Ryan Ybarra, Dionysos Slaga, Jihong Yang, Meric Ovacik, Noël O. Dybdal, Klara Totpal, \\ Melissa R. Junttila, Diego Ellerman, Genee Lee, Mark S. Dennis, Rodney Prell, and Teemu T. Junttila \\ Genentech Inc., South San Francisco, California, USA.
}

\begin{abstract}
Systemic cytokine release and on-target/off-tumor toxicity to normal tissues are the main adverse effects limiting the clinical utility of T cell-redirecting therapies. This study was designed to determine how binding affinity for CD3 and tumor target HER2 impact the efficacy and nonclinical safety of anti-HER2/CD3 T cell-dependent antibodies (TDBs). Affinity was found to be a major determinant for the overall tolerability. Higher affinity for $\mathrm{CD} 3$ associated with rapidly elevated peripheral cytokine concentrations, weight loss in mice, and poor tolerability in cynomolgus monkeys. A TDB with lower CD3 affinity was better tolerated in cynomolgus monkeys compared with a higher CD3-affinity TDB. In contrast to tolerability, T cell binding affinity had only limited impact on in vitro and in vivo antitumor activity. High affinity for HER2 was critical for the tumorkilling activity of anti-HER2/CD3 TDBs, but higher HER2 affinity also associated with a more severe toxicity profile, including cytokine release and damage to HER2-expressing tissues. The tolerability of the anti-HER2/CD3 was improved by implementing a dose-fractionation strategy. Fine-tuning the affinities for both the tumor target and CD3 is likely a valuable strategy for achieving maximal therapeutic index of $\mathrm{CD} 3$ bispecific antibodies.
\end{abstract}

Authorship note: KS and CLZdZ contributed equally to this work.

Conflict of interest: KS, LKS, VC, FZ, MH, RC, JL, J), JL, RY, DS, JY, MO, NOD, KT, MRJ, DE, GL, RP, and TTJ are employees of Genentech Inc., a member of the Roche Group, and hold stock and options.

Copyright: (c) 2020, American Society for Clinical Investigation.

Submitted: September 24, 2019

Accepted: March 11, 2020

Published: April 9, 2020

Reference information: $/ C I$ Insight 2020;5(7):e133757.

https://doi.org/10.1172/jci.

insight.133757.

\section{Introduction}

HER2 is a clinically validated target for multiple monoclonal antibodies, small molecule tyrosine kinase inhibitors, and the antibody-drug conjugate ado-trastuzumab emtansine (1). HER2 is a strong oncogenic driver of tumor cell growth due to overexpression or activating mutations. HER2 gene amplification and/or overexpression occurs in about $20 \%$ of breast cancers (HER2 ${ }^{+}$cancers) and is associated with more-aggressive disease (2), whereas activating mutations are more rare (3). We have generated a highly efficacious antiHER2/CD3 T cell-dependent antibody (TDB) for treatment of HER2 ${ }^{+}$cancer that demonstrates robust potency in HER2-overexpressing tumor models $(4,5)$. T cell-retargeting approaches, such as CAR T cells and CD3-bispecific antibodies, have shown promising response rates and durability of response in the treatment of hematological malignancies (CD19 in B cell malignancies, refs. 6-11; BCMA in multiple myeloma, ref. 12), generating broad excitement for these therapies.

A key challenge for developing $\mathrm{T}$ cell-retargeting therapies for the treatment of solid tumors is that, unlike in hematological indications, tumor-specific targets are not readily available for solid tumor indications. This can result in adverse effects caused by $\mathrm{T}$ cell reactivity to normal tissues that express the antigen. Clinical and preclinical on-target toxicities have been widely reported for engineered $\mathrm{T}$ cell approaches and CD3-bispecific therapies targeting multiple solid tumor antigens, such as CEA, EpCam, gp100, MAGE-A3, and EGFR and HER2 (13-18). HER2 is expressed on epithelial cells in multiple normal tissues, including gastrointestinal, respiratory, reproductive, and urinary tract, as well as in the skin, breast, and placenta (19). The hypothesis underlying the therapeutic index of the anti-HER2/CD3 TDB relies on differential sensitivity of HER2 $2^{+}$tumors and normal tissues, based on HER 2 expression levels $(4,20)$. However, therapeutics redirecting $\mathrm{T}$ cell activity to HER2 ${ }^{+}$tumors tested in clinical trials have illustrated the apparent risk of on-target/off-tumor adverse effects for this target $(14,17)$.

Currently, very little information is available on how key properties, such as affinity to T cells and tumor 
A

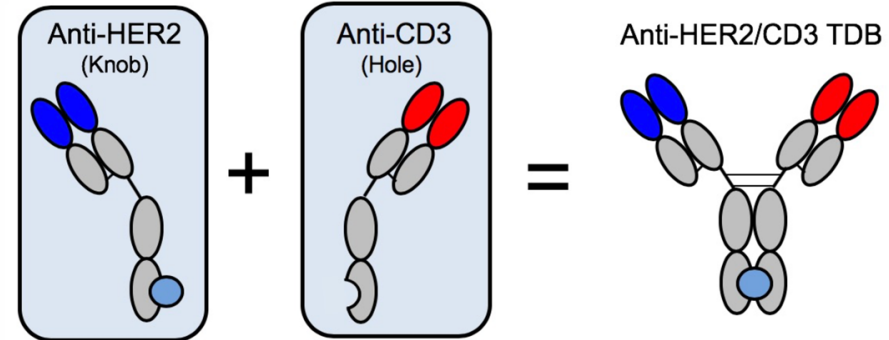

B - HER2-TDB 1 (Lower CD3 affinity) HER2-TDB 2 (Higher CD3 affinity)

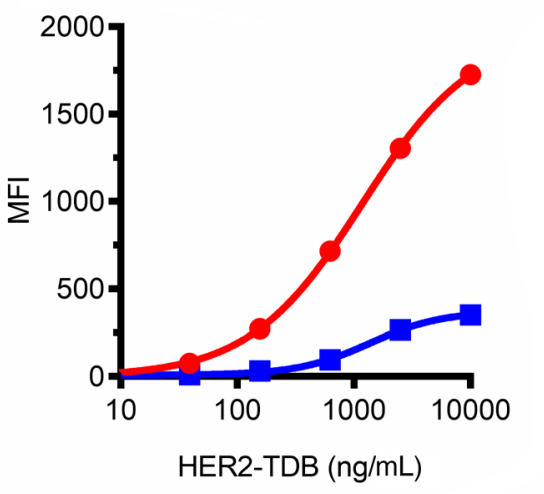

C
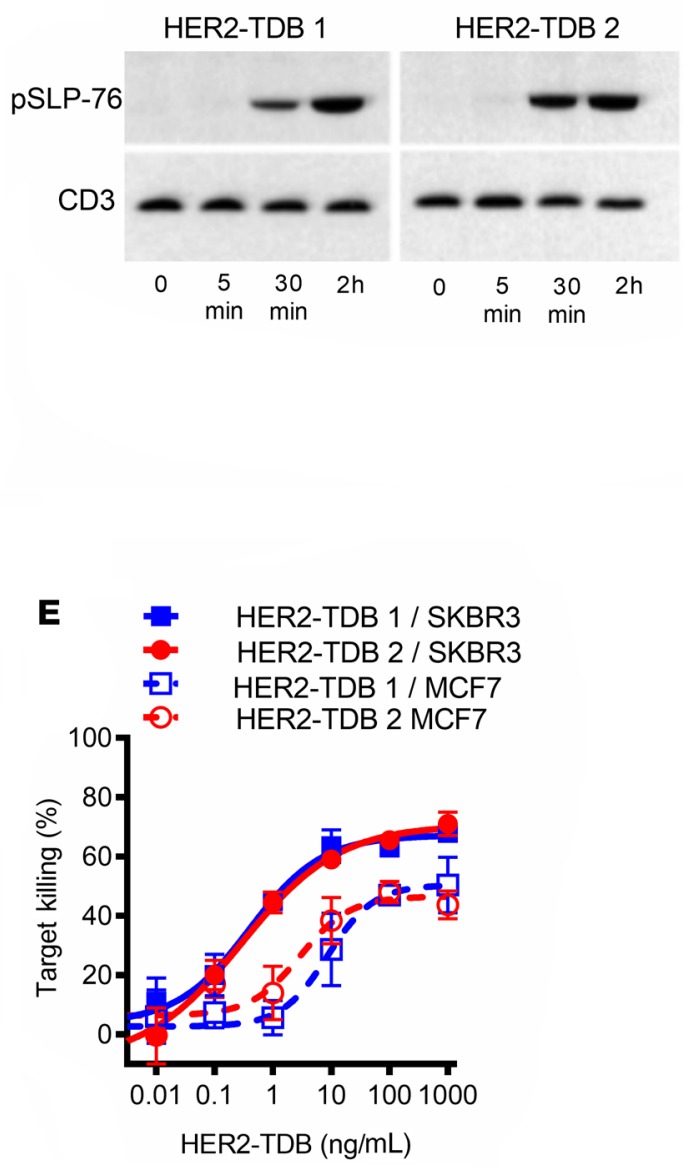

\section{D}
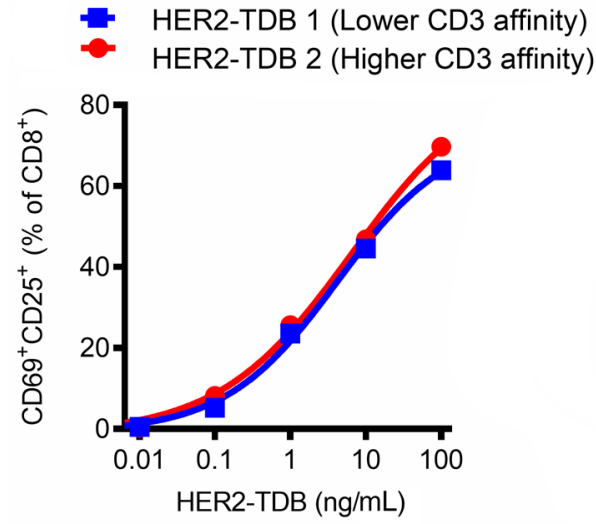

Figure 1. CD3 affinity does not impact in vitro activity of anti-HER2/CD3 TDB. (A) Representation of anti-HER2/ CD3 TDB. (B) Binding to human CD8 ${ }^{+}$ cells was analyzed by flow cytometry ( $n=1)$. (C) TDB-induced (1 $\mu \mathrm{g} / \mathrm{mL}$ TDBs) TCR signaling pathway activation in splenic $\mathrm{CD} 8^{+}$cells extracted from huCD3 transgenic mice was analyzed by phos-SLP-76 Western blot (see complete unedited blots in the supplemental material; lanes were run on the same gel but were noncontiguous; $n=1)$. (D) TDB-induced activation of human CD8 ${ }^{+}$cells was analyzed by flow cytometry $(n=1)$. (E) In vitro killing activity of HER2-amplified SKBR3 (solid lines) and low-HER2-expressing MCF7 (dotted lines) cells was analyzed by Cell Titer Glo viability assay $(n=3)$. Data presented as mean \pm SD.

target, impact the antitumor activity and tolerability of CD3-bispecific antibodies. In this study, we describe the importance of customization of the affinity for both tumor and $\mathrm{T}$ cell antigens to achieve the desired balance between antitumor activity and safety. We further demonstrate that the tolerability of an anti-HER2/ CD3 TDB can be improved by a dose-fractionation strategy. The affinity-optimized anti-HER2/CD3 TDB is able to selectively target HER2-overexpressing tumors in a 2-tumor mouse model, suggesting that a therapeutic index can be achieved for an anti-HER2/CD3 TDB in the treatment of HER2 ${ }^{+}$cancer.

\section{Results}

CD3 affinity does not impact in vitro activity of anti-HER2/CD3 TDB. Anti-HER2/CD3 TDBs (Figure 1A; see below) demonstrate robust single-agent antitumor activity in HER2-overexpressing tumor models (4). To investigate the impact of affinity of target binding on preclinical efficacy and safety, bispecific antibodies with varying affinities for either HER2 or CD3 were created. BIAcore (Table 1) and CD8 ${ }^{+}$cell binding (Figure 1B) consistently demonstrated an approximately 30-fold difference between HER2-TDB 1 (lower CD3 affinity) and HER2-TDB 2 (higher CD3 affinity). CD3 binding affinities were generally substantially lower compared with previous reports (21), likely due to different experimental parameters (e.g., BIAcore mea- 
Table 1. BIAcore affinities $\left(37^{\circ} \mathrm{C}\right)$ of anti-HER2/CD3 TDB variants

\begin{tabular}{|c|c|c|c|c|}
\hline \multirow[t]{2}{*}{ Antibody } & \multicolumn{2}{|c|}{ CD3 $K_{D}(n M \pm S D)$} & \multicolumn{2}{|c|}{ HER2 $K_{\mathrm{D}}(\mathrm{nM} \pm \mathrm{SD})$} \\
\hline & Human & Cynomolgus monkey & Human & Cynomolgus monkey \\
\hline HER2-TDB 1 & $446 \pm 46$ & $387 \pm 36$ & $1.48 \pm 0.08$ & $17.3 \pm 1.6$ \\
\hline HER2-TDB 2 & $14.4 \pm 0.42$ & $12.8 \pm 0.70$ & $1.80 \pm 0.18$ & $19.3 \pm 1.9$ \\
\hline HER2-TDB 3 & $481 \pm 62$ & $534 \pm 23$ & $0.40 \pm 0.17$ & $2.42 \pm 0.11$ \\
\hline
\end{tabular}

surements made at $37^{\circ} \mathrm{C}$ vs. room temperature). HER2-TDB 2 resulted in slightly more robust activation of $\mathrm{T}$ cell receptor signaling compared with HER2-TDB 1, as indicated by phosphorylation of lymphocyte cytosolic protein 2 (phos-SLP-76) (Figure 1C). However, when analyzed by flow cytometry, T cell activation mediated by HER2-TDB 1 and HER2-TDB 2 was comparable (Figure 1D). Similarly, the ability to mediate killing of HER2-amplified SKBR3 cells and MCF7 cells (low HER2 expression, similar to human normal tissue) was nearly identical (Figure 1E). These results indicate that low CD3 affinity is sufficient to induce robust $\mathrm{T}$ cell activation and TDB-mediated killing in the context of targeting the membrane proximal region of HER2 and that increasing CD3 affinity by approximately 30-fold does not enhance $\mathrm{T}$ cell activation or tumor cell killing in vitro.

CD3 affinity does not affect in vivo antitumor activity of anti-HER2/CD3 TDB. The effect of CD3 affinity on antitumor activity of the HER2 TDB was evaluated in a mouse xenograft model. NOD-Prkdc ${ }^{\text {scid }} I L 2 \operatorname{rg}^{\text {null }}$ (NSG) mice supplemented with human peripheral blood mononuclear cells (PBMCs) were engrafted with HER2-amplified KPL4 tumors and administered a single dose of HER2-TDB 1 or HER2-TDB 2. Antitumor activity was comparable for both molecules over the whole dose range that was tested $(0.01-0.5 \mathrm{mg} / \mathrm{kg}$ ) (Figure $2 \mathrm{~A}$ ). The PBMC-engrafted mouse model used in the experiment has limitations, since the biodistribution of therapeutic antibodies can be anomalous in highly immunocompromised mice (22) and humanizing the immune system using i.p. grafted human PBMCs is not fully representative of the intact murine immune system (23). We therefore further evaluated the efficacy and pharmacodynamic (PD) response of anti-HER2/CD3 TDBs in human CD3 transgenic mice (huCD3) (24) crossbred with human HER2 transgenic mice (MMTVhuHER2) (25). This model allows the assessment of human CD3-binding TDBs in a mouse tumor model with an intact immune system. Since human IgG1 is immunogenic in mice, only short-term experiments were performed. Both HER2-TDB 1 and HER2-TDB 2 induced rapid regression of spontaneous HER2+ MMTV tumors (Figure $2 \mathrm{~B}$ ) with no significant difference in the magnitude or incidence of tumor responses. Next, we analyzed the activation and proliferation status of tumor-infiltrating $\mathrm{T}$ cells 6 days after single-dose administration of 0.25 or $0.5 \mathrm{mg} / \mathrm{kg}$ of HER2-TDB 1 or HER2-TDB 2. Both molecules induced increased T cell activation (indicated by $\mathrm{CD}^{+} \mathrm{PD} 1^{+}$) and $\mathrm{T}$ cell proliferation (indicated by $\mathrm{CD} 8^{+} \mathrm{Ki} 67^{+}$) in tumors (Figure $2 \mathrm{C}$ ). A significant difference between HER2-TDB 1 and HER2-TDB 2 was evident at the $0.5 \mathrm{mg} / \mathrm{kg}$ dose level (Figure 2C). In conclusion, both CD3 affinity variants induce robust tumor regression and $\mathrm{T}$ cell activation in vivo at low dose levels. Consistent with the in vitro results (Figure 1), increased affinity for $\mathrm{T}$ cells did not result in substantial improvement of in vivo potency. Despite the detected differences in $\mathrm{T}$ cell activation, taken together, our results consistently demonstrate that both molecules induce strong $\mathrm{T}$ cell activation and tumor cell killing both in vitro and in vivo.

CD3 affinity is associated with systemic cytokine release and weight loss in mice. To evaluate anti-HER2/CD3 TDB-induced in vitro cytokine release, MCF7 cells were incubated with healthy donor whole-blood samples and anti-HER2/CD3 TDB affinity variants. No cytokines were detected in the growth media in the absence of HER2-expressing target cells. HER2-TDB 1 and HER2-TDB 2 both induced dose-dependent increases in IL-2, IL-6, IFN- $\gamma$, and TNF- $\alpha$ (Figure 3A), but cytokine levels were consistently higher for HER2-TDB 2 (higher CD3 affinity) at all concentrations tested.

The cytokine response was evaluated in vivo following administration of a single dose $(0.5 \mathrm{mg} / \mathrm{kg})$ of HER2-TDB 1 or HER2-TDB 2 to MMTVhuHER2.huCD3.TG double transgenic mice bearing spontaneous HER2-overexpressing mammary tumors. Maximal cytokine levels were greater in animals dosed with HER2-TDB 2 (Figure 3B). In animals administered with HER2-TDB 2, IL-2, IL-6, and TNF- $\alpha$ were increased 2 hours after dose. The induction of IFN- $\gamma$ was less pronounced but detectable in a subset of animals 6 hours after dose. All measured cytokines recovered to predose background levels by 24 hours after dose. In addition to increased systemic cytokine release, weight loss was observed in mice in both 
A

051015202530
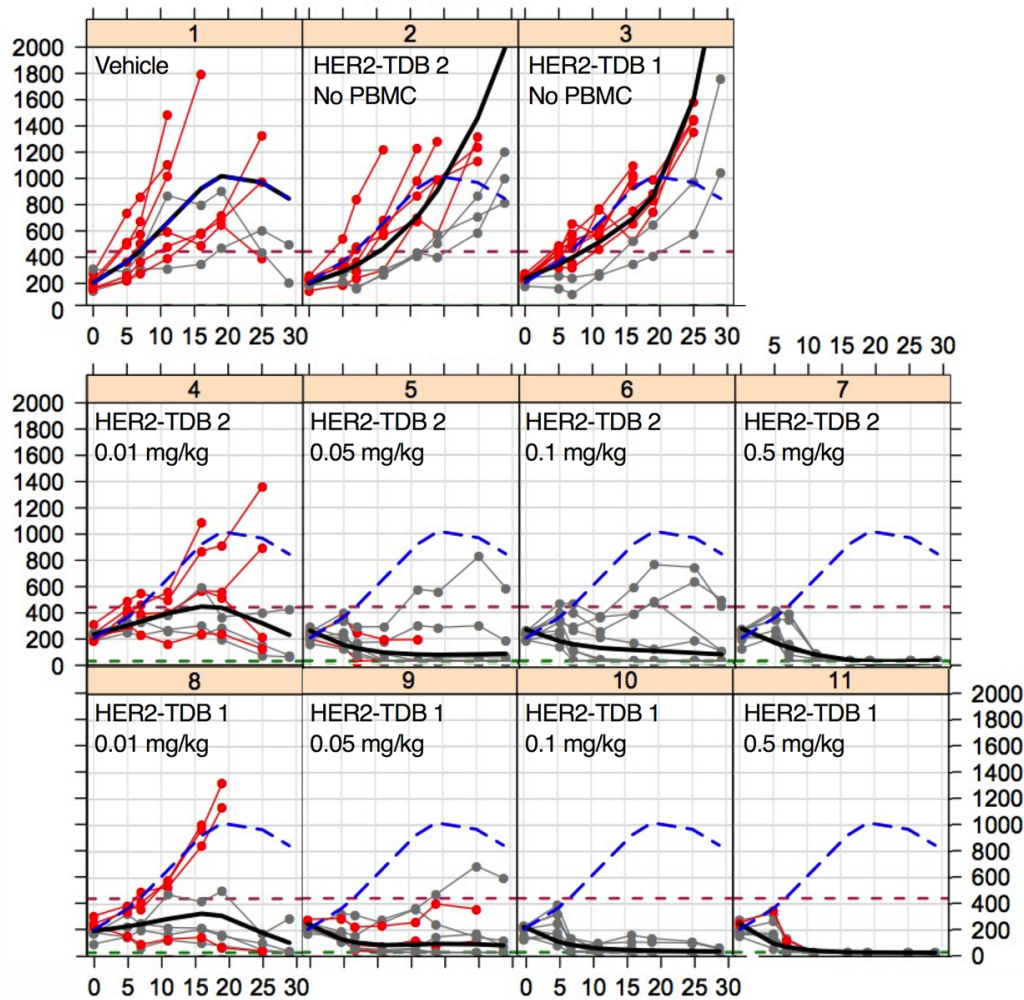

B
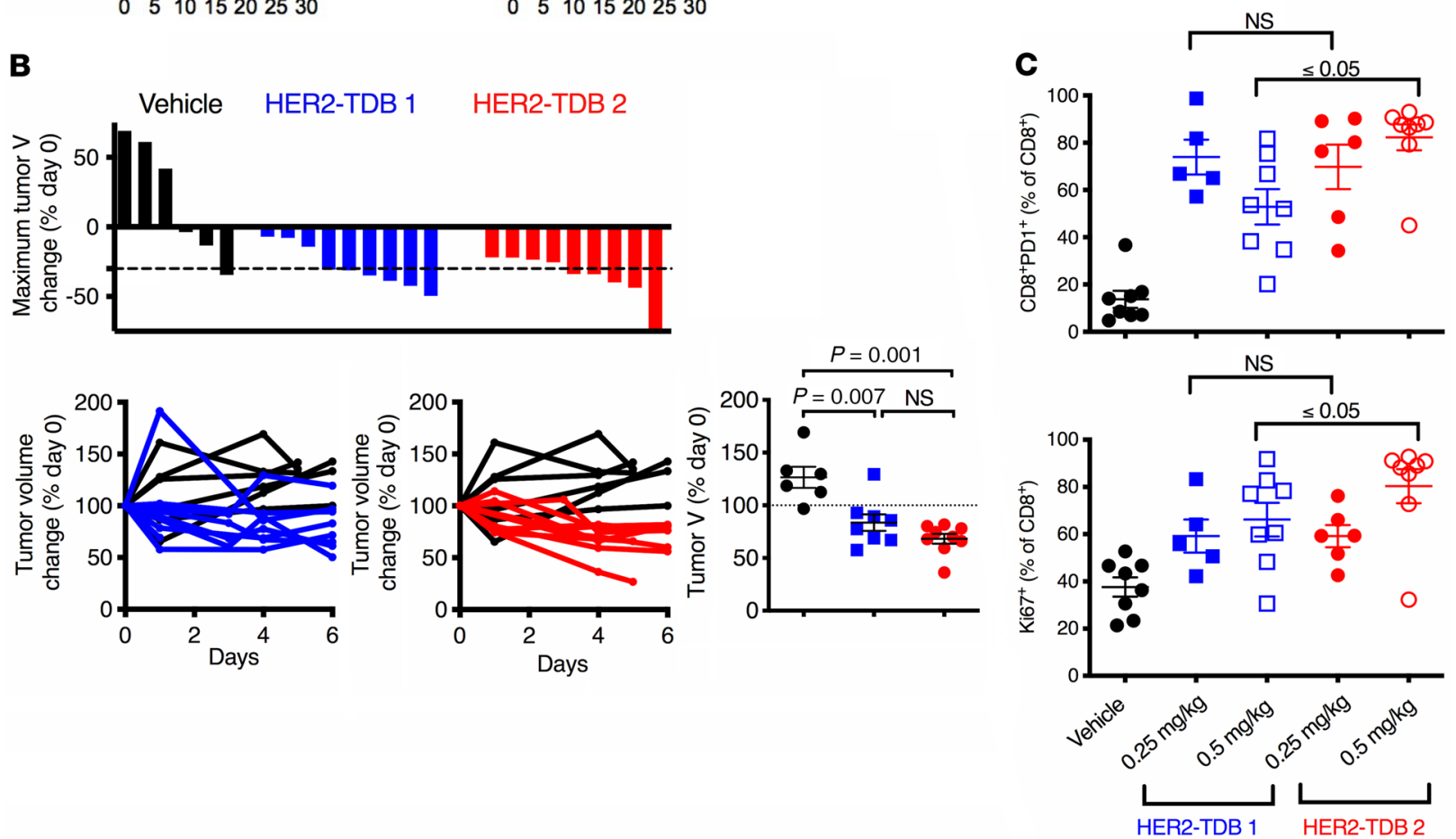

Figure 2. CD3 affinity does not affect in vivo activity of anti-HER2/CD3 TDB. (A) Individual tumor volume response of HER2-amplified KPL4 breast cancer xenografts to HER2-TDB 2 (higher CD3 affinity; groups 4-7) and HER2-TDB 1 (lower CD3 affinity; groups 8-11) in NSG mice supplemented with human PBMCs. Mice with established tumors received a single i.v. dose at day 0 at indicated dose levels. Trellis plots of individual and fitted tumor volumes are presented, with study day on the $x$ axis and tumor volume on the $y$ axis. Each panel in the trellis depicts 1 dose group (panel headers indicate group numbers). Bold, solid black lines indicate the fitted tumor volume for each dose group. Dashed blue lines indicate the fitted tumor volume for the control group (vehicle, histidine buffer). Gray lines indicate the tumor response over time in individual animals present through the course of the study. Red lines indicate the tumor response over time in mice that were removed from the study due to tumor progression beyond prespecified limits. $n=8-9$ for each treatment group. (B and C) Tumor-bearing MMTVhuHER2. huCD3.TC mice were treated with a single dose of HER2-TDB 2 (red), HER2-TDB 1 (blue), or vehicle (black) at day 0. (B) Effect of $0.5 \mathrm{mg} / \mathrm{kg}$ anti-HER2/CD3 TDB on tumor growth. $n=6-11$ for each treatment group. (C) Effect of $0.25 \mathrm{mg} / \mathrm{kg}$ (filled symbols) or $0.5 \mathrm{mg} / \mathrm{kg}$ (open symbols) dose on T cell activation and proliferation markers on tumor-infiltrating CD8+ cells analyzed by flow cytometry 6 days after dose. Error bars represent mean \pm SEM. $n=5-8$ for each treatment group. Statistical analysis using unpaired $t$ test with Welch's correction. 
A

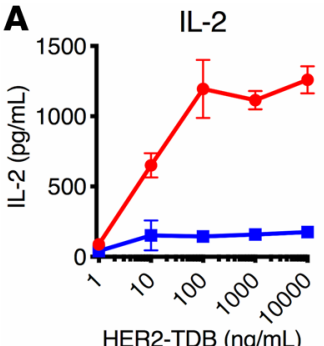

B

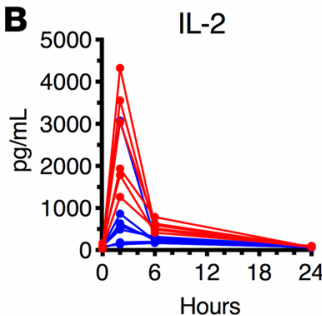

C

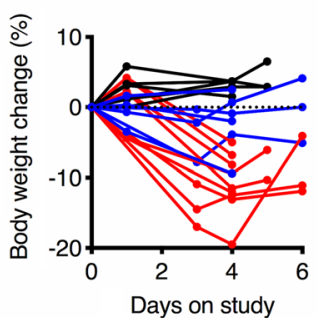

D

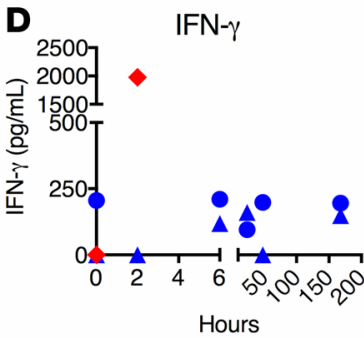

IL-6

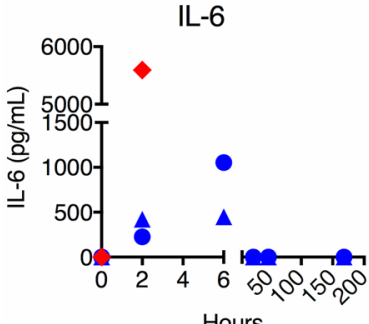

IL-6

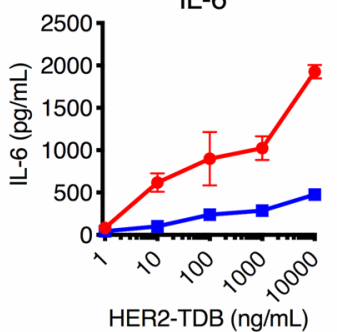

IL-6
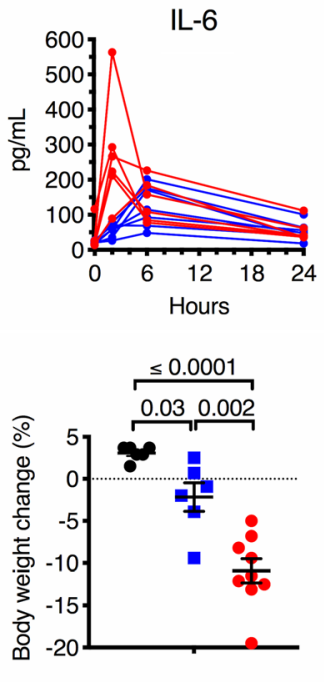

IFN- $\gamma$

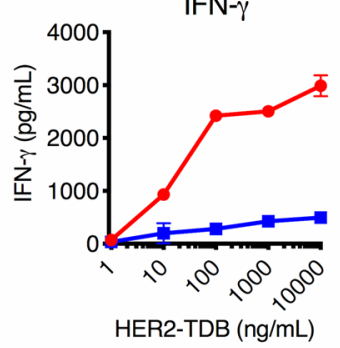

IFN- $\gamma$
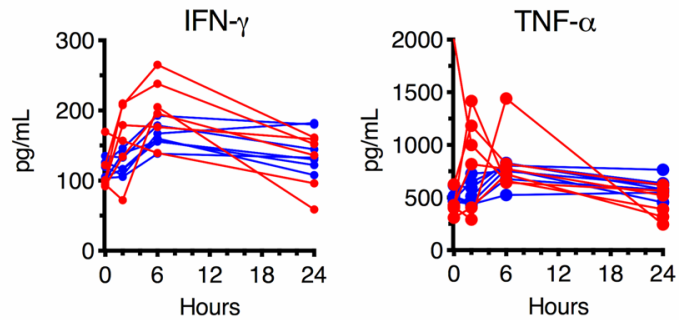

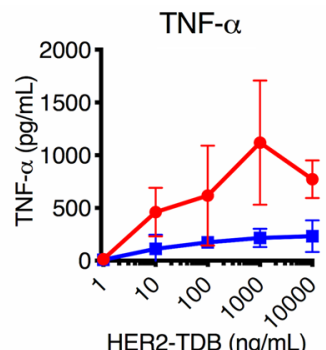

HER2-TDB (ng/mL)
HER2-TDB 1

HER2-TDB 2

Vehicle
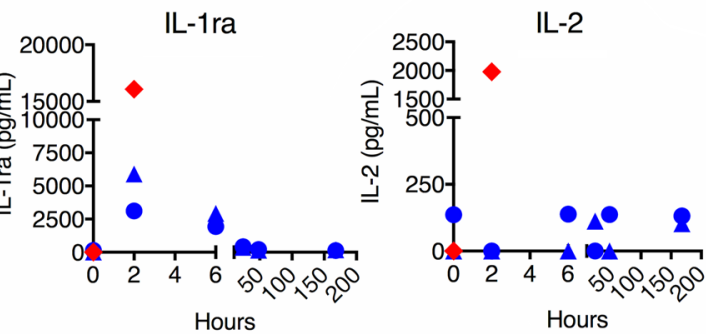

- HER2-TDB 1 (\#1501)

A HER2-TDB $1(\# 1502)$

- HER2-TDB 2 (\#3501)

Figure 3. CD3 affinity is associated with elevated cytokine release and weight loss. (A) To analyze cytokine release in vitro, human healthy donor whole blood was cocultured with MCF7 cells and HER2-TDB 1 (lower CD3 affinity; blue) or HER2-TDB 2 (higher CD3 affinity; red) for 20 hours, and cytokine levels in media were analyzed by Bio-Plex Pro Human Cytokine Assay. Data presented as mean $(n=3) \pm$ SD. (B and C) Tumor-bearing MMTVhuHER2.huCD3.TC mice were treated with $0.5 \mathrm{mg} / \mathrm{kg}$ dose of indicated anti-HER2/CD3 variant $(n=6-7)$. (B) Serum cytokines were analyzed at indicated time points by Luminex. (C) Body weight change after TDB treatment $(n=6-9)$. Statistical analysis (unpaired $t$ test with Welch's correction) of body weights 4 days after treatment are presented in the right panel. Error bars represent mean \pm SEM. (D) In vivo cytokine levels from cynomolgus monkeys treated with a single dose of $0.5 \mathrm{mg} / \mathrm{kg}$. Data points represent individual animals $(n=2$ for TDB $1 ; n=1$ for TDB 2 ). 
TDB variant groups (Figure 3C) for 6 days after treatment. The weight loss was significantly greater in mice treated with HER2-TDB 2. Neither molecule resulted in body temperature changes (data not shown).

Impact of CD3 affinity on PD and tolerability of anti-HER2/CD3 TDBs in cynomolgus monkeys. To assess how CD3 affinity impacts the nonclinical safety profile in vivo, a single-dose toxicity study was conducted using HER2-TDB 1 and HER2-TDB 2 in cynomolgus monkeys at a dose level of $0.5 \mathrm{mg} / \mathrm{kg}$ administered via slow bolus i.v. injection.

HER2-TDB 1 was well tolerated, with transient and generally reversible clinical pathology (clinical chemistry and hematology) changes observed. Two animals were observed for 7 days after dose with out notable adverse effects. Treatment resulted in early transient decreases in lymphocytes (Supplemental Figure 1A; supplemental material available online with this article; https://doi.org/10.1172/jci. insight.133757DS1); 2-6 hours after dose, MCP-1, IL-1 receptor antagonist (IL-1RA), and IL-6 were elevated (Figure 3D) and T cell activation markers (CD69/CD25) were increased (data not shown). Clinical chemistry changes included minimal to mild increases in alanine aminotransferase (ALT), aspartate aminotransferase (AST), alkaline phosphatase (ALP), bilirubin, and fibrinogen and a decrease in albumin (Supplemental Figure 1B). All clinical chemistry parameters trended toward or approximated baseline values by the end of the study. TDB-related histopathology findings at necropsy, which was performed 7 days after single-dose administration, were limited to minimal portal mixed cell infiltrates in the liver and minimal increased lymphoid cellularity in the spleen in 1 animal each.

In contrast, HER2-TDB 2 was not tolerated $(n=1)$ at the $0.5 \mathrm{mg} / \mathrm{kg}$ dose level and resulted in early euthanasia on the day of dosing. High levels of systemic proinflammatory cytokines including IFN- $\gamma$, TNF- $\alpha$, IL-2, IL-6, MCP-1, and IL-1RA (Figure 3D) were detected 2 hours after dose administration, consistent with cytokine release syndrome (CRS). Clinical pathology evaluation was not performed in this animal; however, findings noted at necropsy included microscopic inflammatory cell infiltrates in multiple organs (neutrophilic or mixed inflammatory cell infiltrates in the liver, adrenal gland, and mesenteric lymph node) consistent with a systemic inflammatory response.

In summary, HER2-TDB 1 was well tolerated in cynomolgus monkeys at a dose of $0.5 \mathrm{mg} / \mathrm{kg}$, but HER2TDB 2 was not tolerated. Increasing the affinity for CD3 was associated with severe adverse effects and systemic cytokine release. The distribution and character of findings in the animal dosed with HER2-TDB 2 were largely consistent with expected tissue HER2 expression and pharmacology of the anti-HER2/CD3 TDBs.

HER2 affinity affects the killing activity of anti-HER2/CD3 TDBs. The effect of HER2 affinity on in vitro killing activity was tested using affinity variants of trastuzumab (clone 4D5) in conjunction with the lower CD3 affinity arm. The monovalent affinities of the variants for human HER2 have previously been reported as $\mathrm{K}_{\mathrm{D}}$ 0.3, 14, and $49 \mathrm{nM}$ 4D5-WT, 4D5-Y55E.D98A.F100A.Y102A, and 4D5-H91A, respectively (20). High HER2 affinity associated with high in vitro killing activity, suggesting that it is a critical determinant of HER2-TDB potency (Figure 4A) (20). Monovalent binding affinity of 4D5 is approximately 10-fold lower for cynomolgus monkey HER2 compared with human HER2. For studies evaluating the impact of HER2 affinity on preclinical safety, we selected 4D5 and a sequence variant of 4D5 (HER2-TDB 3), which binds to cynomolgus monkey HER2 with 7-fold higher affinity compared with HER2-TDB 1 (Table 1). To demonstrate activity when targeting cynomolgus monkey HER2, CHO cell lines expressing human or cynomolgus monkey HER2 at comparable expression levels were generated (Supplemental Figure 2A). Bivalent 4D5 (trastuzumab) demonstrated similar binding to both cell lines (Supplemental Figure 2B). Cellular binding of the TDB variants to cynomolgus monkey HER2-expressing CHO cells were consistent with measured affinities (Supplemental Figure 2C and Table 1). As expected, HER2-TDB 3 was 10-fold more potent in inducing killing of cynomolgus monkey HER2-expressing CHO cells compared with HER2-TDB 1 (Figure 4B). In summary, these results indicate that HER2 affinity is critical for anti-HER2/ CD3 TDB activity and validate HER2 affinity variants for preclinical safety studies.

Impact of HER2 affinity on pharmacology and tolerability of anti-HER2/CD3 TDBs in cynomolgus monkeys. To investigate the impact of HER2 affinity on in vivo tolerability, a study in cynomolgus monkeys was conducted to compare the HER2 affinity variants at dose levels of 0.5 and $1.5 \mathrm{mg} / \mathrm{kg}(n=4 /$ group) administered via 1-hour i.v. infusion on days 0 and 7.

Administration of HER2-TDB 1 to cynomolgus monkeys at both dose levels was well tolerated, with no observed adverse effects. In contrast, HER2-TDB 3 was not tolerated, resulting in early euthanasia of 1 of 4 animals in both the $0.5 \mathrm{mg} / \mathrm{kg}$-dose and $1.5 \mathrm{mg} / \mathrm{kg}$-dose groups on days 2 and 3 , respectively, based on declining or moribund condition (observations included reduced activity, hunched posture, reduced 

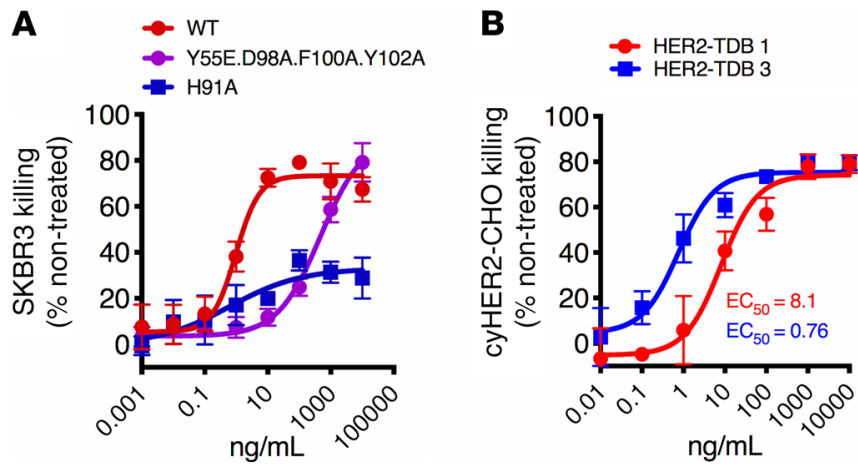

Figure 4. HER2-affinity affects the killing activity of anti-HER2/CD3. (A) SKBR3 cells were treated with HER2 affinity variants of anti-HER2/CD3 TDB. (B) CHO cells were transfected to express cyno-HER2 and treated with HER2-TDB 1 (red) or HER2-TDB 3 (blue). Viability was measured using Cell Titer Glo. Data presented as mean \pm SD $(n=3)$.

appetite, tachycardia, and dyspnea or tachypnea). Furthermore, clinical signs such as emesis and liquid feces/diarrhea were more frequently observed in animals treated with HER2-TDB 3.

Changes in immunophenotyping, hematology, and coagulation parameters were generally of similar magnitude for HER2-TDB 1 and HER2-TDB 3 (Figure 5 and Supplemental Figure 3); however, peripheral $\mathrm{T}$ cell activation and selected cytokines (IL-6, IFN- $\gamma$ ) were more notably elevated after HER2-TDB 3 treatment (Figure 5, A and B). Changes in clinical pathology parameters (Figure 5 and Supplemental Figure 3) were consistent with the expected pharmacologic effect of acute cytokine release leading to an acute-phase inflammatory response and margination of blood lymphocytes. This included decreases in T cells, monocytes, and albumin; increases in neutrophils, fibrinogen, CRP, ALT, AST, ALP, total bilirubin, BUN, and creatinine; and prolongation of activated partial thromboplastin time (Figure 5C and Supplemental Figure 3). These changes were most prominent within first 24 hours and decreased in magnitude following administration of the second dose on day 7 and throughout the follow-up period. Acute, transient cytokine elevations and lymphocyte activation were observed in both the HER2-TDB 1 and HER2-TDB 3 treatment groups (Figure 5B), consisting of increased MCP-1, IL-6, IL-1RA, and IFN- $\gamma$. Reduction of CD4 $4^{+}$and CD8 ${ }^{+} \mathrm{T}$ cells (most notably 6 hours after the first dose) corresponded with increased $\mathrm{T}$ cell activation $\left(\mathrm{CD} 69^{+}\right)$(Figure 5 , A-C). The 2 animals that did not tolerate HER2-TDB 3 demonstrated increased systemic IL- 6 and IFN- $\gamma$ (Figure 5B) compared with the surviving animals in the same dose groups.

Histopathological findings in animals administered HER2-TDB 1 were limited to minimal mononuclear cell infiltrate in the mammary gland of 1 of 4 animals in the $1.5 \mathrm{mg} / \mathrm{kg}$-dose group but were more pronounced in animals administered HER2-TDB 3. Observations in the 2 HER2-TDB 3-dosed animals that underwent early euthanasia included inflammatory cell infiltrates or inflammation mainly in HER2-expressing organs and tissues, exemplified by epithelial single cell necrosis and, occasionally, ulceration in the vagina, mandibular salivary gland, skin, and/or i.v. administration site; alveolar-interstitial inflammation in the lung; tubular damage in the kidney; and increased epithelial mitoses in the skin. In addition, decreased cellularity in the BM and lymphoid organs were observed. Microscopic observations in HER2-TDB 3-dosed animals that survived to scheduled necropsy were similar but generally less severe and less extensive. Most of the TDB-related changes noted at terminal necropsy were not present by the end of the recovery period.

These data demonstrate that HER2-TDB 1 was well tolerated at dose levels up to $1.5 \mathrm{mg} / \mathrm{kg}$ but that the higher-affinity HER2-TDB 3 was not tolerated even at the lower dose of $0.5 \mathrm{mg} / \mathrm{kg}$. In addition, increased affinity to HER2 correlated with more severe histopathologic effects in HER2-expressing tissues.

Dose fractionation improves tolerability of an anti-HER2/CD3 TDB in cynomolgus monkey. HER2-TDB 3 was not tolerated in cynomolgus monkeys following a single 1-hour i.v. infusion of $0.5 \mathrm{mg} / \mathrm{kg}$ or $1.5 \mathrm{mg}$ / $\mathrm{kg}$. Given the comparable affinity of this molecule for human and cynomolgus monkey HER2, it seemed possible that the observations in cynomolgus monkeys may be predictive of the expected tolerability profile of an anti-HER2/CD3 TDB in humans. Therefore, an additional repeat dose study in cynomolgus monkeys was conducted to evaluate whether dose fractionation (i.e., intra-animal dose escalation) could ameliorate the acute cytokine release and poor tolerability observed following administration of HER2-TDB 3.

HER2-TDB 3 was administered via 1-hour i.v. infusion at dose levels of $0.1 \mathrm{mg} / \mathrm{kg}$ or $0.2 \mathrm{mg} / \mathrm{kg}$ on 


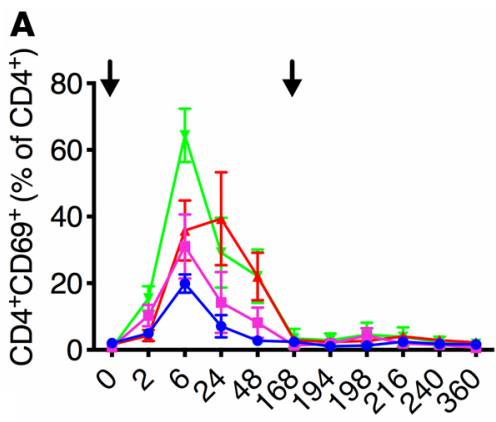

Hours
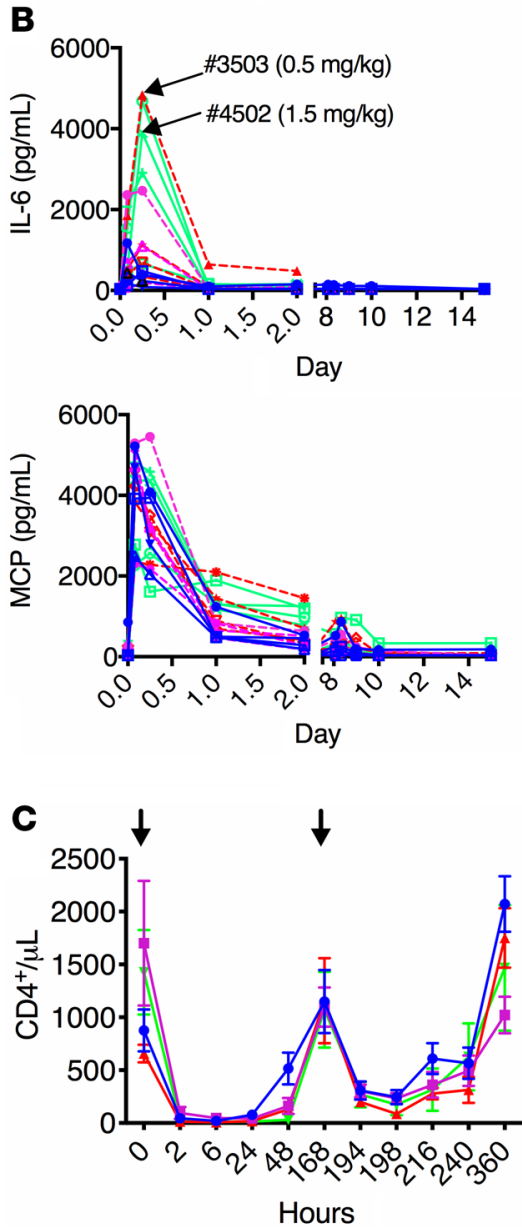

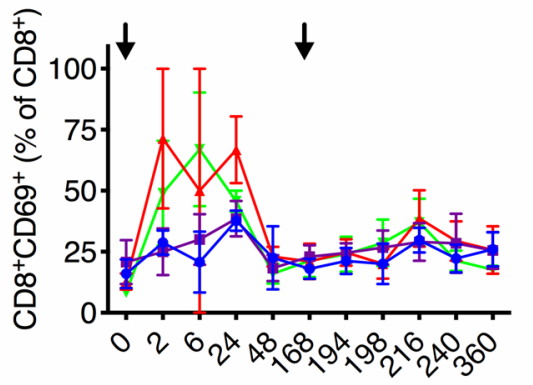

Hours
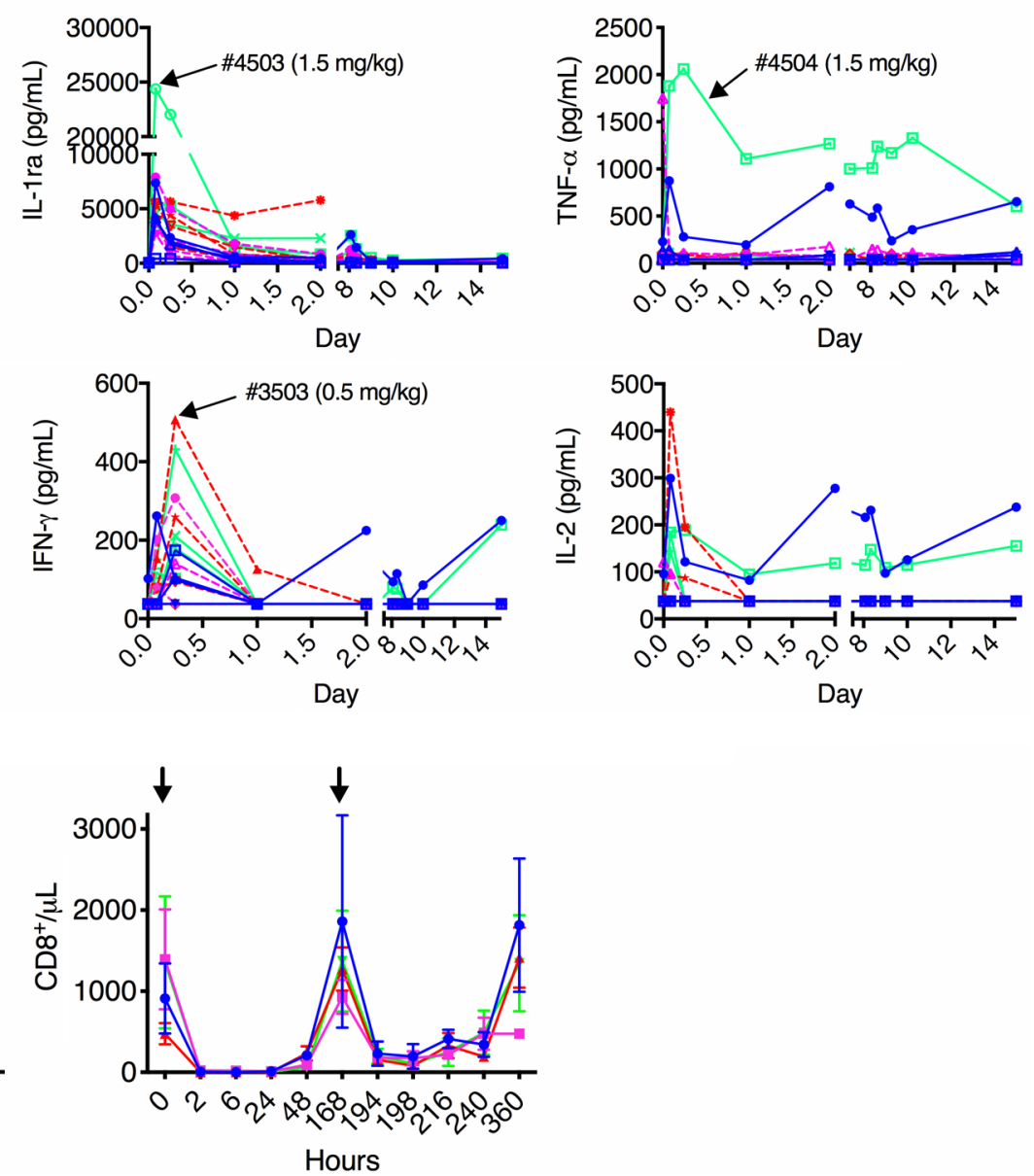

$0.5 \mathrm{mg} / \mathrm{kg}$ HER2-TDB 1

A. $0.5 \mathrm{mg} / \mathrm{kg}$ HER2-TDB 3

$\neq 1.5 \mathrm{mg} / \mathrm{kg}$ HER2-TDB 3
$1.5 \mathrm{mg} / \mathrm{kg} \mathrm{HER} 2-\mathrm{TDB} 1$

Figure 5. Impact of HER2 affinity to anti-HER2/CD3 TDB induced leukocyte changes and acute systemic cytokine release in cynomolgus monkeys. Cynomolgus monkeys were treated with 0.5 and $1.5 \mathrm{mg} / \mathrm{kg}$ of HER2-TDB 1 (lower HER2 affinity; blue and magenta, respectively) or HER2-TDB 3 (higher HER2 affinity; red and green, respectively) on days 0 and 7. (A-C) Peripheral blood was sampled at indicated time points and analyzed for T cell activation (CD69) (A), systemic cytokine levels (B), and number of CD8 ${ }^{+}$and CD4+ lymphocytes (C). Data are presented as mean \pm SEM (A and C) or individual animals (B). Arrowheads indicate time of dosing (A and C) or point out individual animals where dosing was not tolerated (3503 and 4502 ) or tolerated (4503 and 4504) (B). $n=3-4$ for each treatment group.

day $0,0.2 \mathrm{mg} / \mathrm{kg}$ on day 1 , and $0.4 \mathrm{mg} / \mathrm{kg}$ on day $7(n=3)$. Dose fractionation resulted in a reduction in the $\mathrm{C}_{\text {max }}$ after the first dose (Figure 6A and Supplemental Table 1).

HER2-TDB 3 was well tolerated up to $0.4 \mathrm{mg} / \mathrm{kg}$ when the first dose was fractionated over $2 \mathrm{sub}$ sequent days. Furthermore, the acute phase response was attenuated, and no TDB-related clinical signs were noted. Additionally, IL- 6 and IFN- $\gamma$ increases were smaller in magnitude compared with the nonfractionated $0.5 \mathrm{mg} / \mathrm{kg}$ dose (Figure 6B). No TDB-related histopathology findings were observed at necropsy following administration of dose-fractionated HER2-TDB 3. 
A

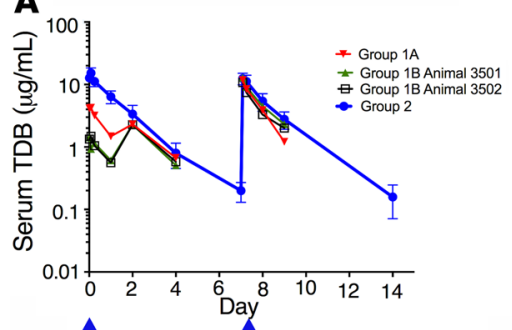

$$
\text { 个个 } 0.4 \uparrow
$$

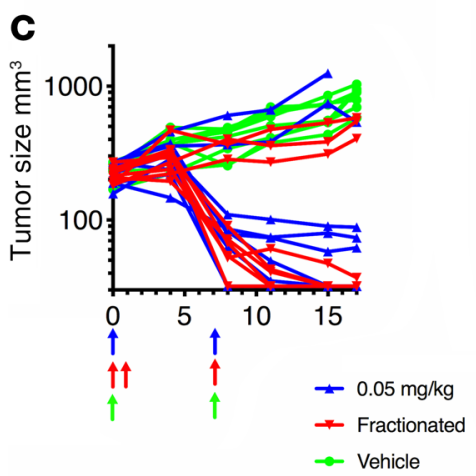

B
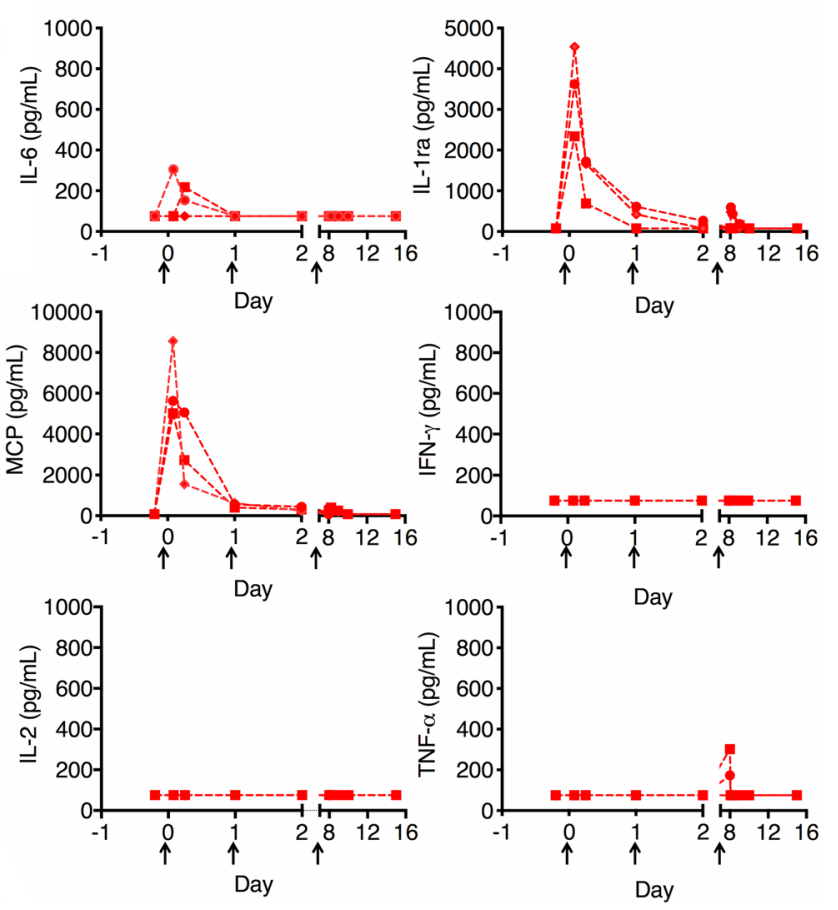

Figure 6. Dose fractionation reduces anti-HER2/CD3 TDB-induced systemic cytokine release in cynomolgus monkey but retains antitumor activity in mice. (A) Effect of dose fractionation of HER2-TDB 3 on systemic exposure in cynomolgus monkey. Animals in group $1 \mathrm{~A}(0.2 \mathrm{mg} / \mathrm{kg}$ on days 0 and $1,0.4 \mathrm{mg} / \mathrm{kg}$ on day $7 ; n=1)$ and group $1 \mathrm{~B}(0.1 \mathrm{mg} / \mathrm{kg}$ on days 0 and $1,0.4 \mathrm{mg} / \mathrm{kg}$ on day $7 ; n=2)$ were dosed using dose fractionation. Animals in group $2(n=4)$ received $0.5 \mathrm{mg} /$ $\mathrm{kg}$ on days 0 and 7. Blood samples were collected at indicated time points, and human IgG was detected by ELISA. Data are presented as mean \pm SD for group 2 and as exposure for individual animal for group 1 . PK parameters are presented in Supplemental Table 1. (B) Serum cytokine analysis using Luminex from cynomolgus monkeys dosed using the dose fractionation strategy ( $n=3$, individual animal data depicted). (C) Individual tumor volume response of HER2-amplified KPL4 breast cancer xenografts to anti-HER2/CD3 TDB in NSG mice supplemented with human PBMCs. Mice were treated with $0.05 \mathrm{mg} / \mathrm{kg}$ (once a week x2) dose (blue). Alternatively, the initial dose was fractionated to 2 doses of $0.025 \mathrm{mg} / \mathrm{kg}$ administered on days 0 and 1 (red). $n=8-9$ for each dose group. Arrows indicate time of dosing.

In summary, these results suggest that dose fractionation can improve the tolerability of HER2-CD3 bispecific antibodies.

A dose-fractionated anti-HER2/CD3 TDB retains antitumor activity. Recognizing that dose fractionation may be a useful tool to mitigate clinical safety concerns, the impact of this dosing regimen on antitumor activity in an in vivo mouse xenograft model was evaluated. Mice with established HER2-amplified KPL4 tumors were dosed weekly with $0.05 \mathrm{mg} / \mathrm{kg}$ of HER2-TDB 1 (Figure 6C). Alternatively, the initial dose of $0.05 \mathrm{mg} /$ $\mathrm{kg}$ was fractionated into two $0.025-\mathrm{mg} / \mathrm{kg}$ doses administered on day 0 and day 1 , followed by weekly doses of $0.05 \mathrm{mg} / \mathrm{kg}$. HER2-TDB 1 was equally active with both dose schedules, demonstrating that dose fractionation can maintain antitumor efficacy while providing an option to mitigate acute toxicity in the clinic.

In vivo selectivity for HER2-overexpressing tumors in mice supports preclinical therapeutic index for an anti-HER2/CD3 TDB. Anti-HER2/CD3 TDBs do not bind to normal mouse tissues, and cynomolgus monkeys do not develop HER2-overexpressing tumors. To test the selectivity of an anti-HER2/CD3 TDB for HER2-overexpressing tumors in a single-animal model in vivo, 2 human xenograft tumors with different levels of HER2 expression were engrafted in opposite flanks of mice (Figure 7A), and a single $0.05 \mathrm{mg} / \mathrm{kg}$ i.v. dose of HER2-TDB 1 was administered. KPL4 is a HER2-amplified tumor xenograft that was used to model HER2-overexpressing tumor. HT55 expresses similar levels of HER2 compared with MCF7 and noncancerous cells, such as cultured cardiomyocytes (4), and was used to model on-target activity on low HER2-expressing tissues. HER2 copy number has been previously determined in KPL4 (>600.000) and HT55 ( 25.000) (20). HER2-TDB 1 induced regression of KPL4 tumors (Figure 7B) but did not impact progression of HT55 tumors, further supporting the hypothesis that therapeutic index based on the high expression level of HER2 in HER2 ${ }^{+}$tumors is attainable. 

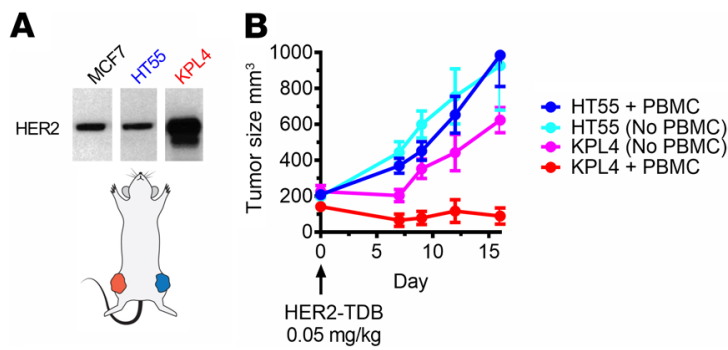

Figure 7. In vivo selectivity to HER2-overexpressing tumors in mice supports therapeutic index for anti-HER2/ CD3 TDB. (A) In the 2-tumor model, each NSG mouse was implanted with HER2-amplified KPL4 tumors and HT55 tumors, which express low levels of HER2. Mice were further supplemented with human PBMCs by i.p. injection. HER2 expression was analyzed by Western blot. Lanes were run on the same gel but were noncontiguous $(n=1)$. (B) Mice with 2 established tumors were treated with single $0.05 \mathrm{mg} / \mathrm{kg}$ dose of HER2-TDB 1. $n=5$ for each group. Data represented as mean $\pm \mathrm{SEM}$.

\section{Discussion}

Optimally, T cell-retargeting therapies can result in robust clinical antitumor activity. Extreme potency of redirected $\mathrm{T}$ cells, however, also poses substantial safety risks. While $\mathrm{T}$ cell-retargeting therapies have been successful in hematological cancers, solid tumor indications have unique challenges, including the lack of lineage-restricted targets expression. Because several of the solid tumor targets also have widespread normal tissue distribution, it is even more paramount to understand how TDB characteristics such as affinity impact both safety and efficacy. In this study, we addressed how binding affinity for tumor and $\mathrm{T}$ cell targets affect properties of an anti-HER2/CD3 TDB. We demonstrated that lower affinities for both antigens were generally better tolerated compared with higher-affinity variants. Antitumor activity, on the other hand, depended on high HER2 affinity, whereas CD3 affinity did not affect the efficacy.

Based on the mechanism of action of anti-HER2/CD3 TDB, key expected toxicities were CRS and T cell-mediated activity on HER2-expressing healthy tissues. Additionally, dose-limiting neurotoxicity has been detected in patients treated with the anti-CD19/CD3 bispecific T cell engager (BiTE) blinatumomab and CD19-chimeric antigen receptor $\mathrm{T}$ cell $(\mathrm{CAR} T)$ constructs $(6,26)$. As the mechanism of neurotoxicity of these agents is largely unclear, it is also a potential risk for an anti-HER2/CD3 TDB.

Cynomolgus monkey toxicity studies with anti-HER2/CD3 TDBs revealed a robust and expected pharmacologic effect, including acute cytokine release, an acute-phase inflammatory response, and peripheral lymphocyte margination. Most TDB-induced findings were transient and reversible and included clinical signs and changes in serum chemistry consistent with CRS (e.g., emesis, liquid feces, reduced activity, and increased serum cytokines and CRP). The US Food and Drug Administration (FDA) defines CRS in nonclinical in vivo safety models by evidence of increased levels of cytokines and at least 3 CRS-related clinical findings, including emesis, diarrhea, tremor, hypoactivity/lethargy, redness or swelling of face, body temperature change, hypotension, heart rate change, or dyspnea in a dose group (27). Adverse effects induced by HER2-TDB 2 and HER2-TDB 3 at the $0.5 \mathrm{mg} / \mathrm{kg}$-dose level meet the CRS criteria.

Retargeting T cells to kill HER2-expressing cells may induce adverse effects on HER2-expressing tissues at high dose levels. Tissue damage was detected in multiple tissues following administration of HER2TDB 3; with the exception of the liver, all affected tissues express HER2. Differentiating between direct T cell activity on tissue and secondary toxicity caused by inflammation and cytokines can be difficult, and the hepatic findings may potentially be secondary to cytokine-induced hepatocellular damage and related immune cell infiltration.

None of the anti-HER2/CD3 TDB constructs tested induced histopathological findings in the central nervous system in our studies. However, some of the clinical symptoms of neurotoxicity such as confusion, aphasia, speech disorders, and dizziness cannot be evaluated in preclinical safety models.

HER2 is expressed in cardiomyocytes, and cardiac dysfunction is a recognized clinical adverse effect of HER2-targeted therapies (28). No cardiovascular effects were observed in our studies; however, minimal multifocal mononuclear cell infiltrates in the heart and myocardial degeneration were noted in individual monkeys. Since these histologic findings are recognized background findings in cynomolgus monkeys, it is unclear whether these findings were related to anti-HER2/CD3 TDB administration (29). Evidence from previous nonclinical studies suggests that anti-HER2 therapy may not be directly cardiotoxic but rather can interrupt 
important cardioprotective functions of the HER2 signaling pathway in the damaged heart, interfering with cardiac myocytes survival and repair of injury (30). Although no evidence of overt cardiotoxicity was detected in these studies, it is recognized that nonclinical animal models are suboptimal predictors of the cardiotoxicity observed clinically with HER2-targeting biologics (31). Therefore, the inclusion of cardiac function monitoring in clinical trials with anti-HER2/CD3 TDBs may be appropriate as a conservative safety measure.

The clear difference in dependency on high HER2 affinity but not on high CD3 affinity for anti-HER2/ CD3 activity was a somewhat unexpected finding. The membrane proximal epitope on HER2 appears to facilitate an effective interaction between tumor cells and T cells, supporting our previous studies (32) and illustrating that the optimal binding properties to tumor cells are critical determinants for high activity of a bispecific antibody. In context of optimal target cell binding, T cell affinity is not rate limiting for triggering and lysis of target cells. Naturally, target biology (e.g., copy number, structural features, and localization in membrane microdomains) is likely to play a significant role in affinity dependencies, and our findings may not accurately predict what the drivers are in other contexts. We have previously demonstrated that high T cell affinity perturbs HER2-dependent distribution of an anti-HER2/CD3 TDB to the tumor - driving it, instead, to secondary lymphatic organs (33); therefore, the comparable in vivo efficacy of HER2-TDB 1 and HER2-TDB 2 was somewhat unexpected. However, in vitro studies have demonstrated that very low receptor occupancy $(0.01 \%-0.05 \%)$ is sufficient for anti-HER2/CD3 TDB-mediated T cell triggering (4), consistent with studies of TCR triggering and suggesting that engagement of as few as 1-25 TCRs is sufficient for $\mathrm{T}$ cell responses (34-36).

In contrast to efficacy, the safety profile of the anti-HER2/CD3 TDBs was substantially affected by $\mathrm{T}$ cell affinity. Higher-affinity CD3 binding resulted in higher levels of cytokine release in vitro, in mice, and in cynomolgus monkeys. Furthermore, high cytokine levels were associated with weight loss in mice and serious adverse effects in cynomolgus monkey safety studies. In general, impacts of affinities on activity and safety of CD3 bispecific molecules are likely target biology- and context-dependent properties, and caution should be used in extrapolating from our studies. However, higher CD3 affinity has been previously reported to associate with elevated cytokine production and reduced tolerability in the context of hematological targets CLL-1 (21) and CD38 (37), extending our findings and suggesting more generalizable risk associated with high CD3 affinity.

High HER2 affinity is critical for the antitumor activity of an anti-HER2/CD3 TDB (Figure 4) (20). However, higher affinity to the tumor antigen is also correlated with a more severe adverse effect profile, clearly demonstrating that increasing the potency of the molecule comes with the trade-off of increased risk for adverse effects. HER2-TDB 3 was poorly tolerated and induced substantial microscopic findings in multiple HER2-expressing tissues, including apoptosis, tissue damage, and inflammatory infiltrates in multiple epithelial tissues. The development of adverse effects occurred at different time points with HER2TDB 2 and HER2-TDB 3, suggesting that the mechanisms of toxicity may differ depending on the affinity to each targeting arm of the molecule.

Nonidentical binding affinity to either tumor antigen or CD3 between human and the nonclinical toxicology species is a common challenge for modeling the nonclinical safety of CD3 bispecific constructs. Differences in affinity should be considered when interpreting nonclinical safety studies with molecules that differ substantially in affinity between human and the toxicology species.

Dose fractionation, also called step dosing, is a recognized strategy for the mitigation of cytokine release and associated toxicities following administration of blinatumomab (38). Data from the cynomolgus monkey study of HER2-TDB 3 in which the $0.5 \mathrm{mg} / \mathrm{kg}$ single dose was fractionated into 2 sequential $0.2-\mathrm{mg} / \mathrm{kg}$ doses transformed a nontolerated dose to one that was well tolerated, confirming that this strategy may be broadly applicable to the mitigation of adverse events with CD3-bispecific molecules and suggesting that $\mathrm{C}_{\max }$ may be a major driver of $\mathrm{T}$ cell-engaging bispecific antibody-mediated acute toxicities. In vivo efficacy was not impaired by dose fractionation, suggesting that efficacy may be more dependent on sustained exposure. Further studies are required to understand the long-term tolerability of anti-HER2/CD3 TDBs.

Our data indicate that affinity is a critical determinant of both efficacy and safety, and demonstrates the importance of detailed nonclinical safety and efficacy studies that enable optimization of the efficacy/ safety profile for bispecific molecules prior to clinical trials. Our nonclinical studies also served to identify adverse effect risks that require clinical monitoring and management. Finally, we confirmed that intrapatient dose escalation (dose fractionation/step dosing) may be an effective and simple strategy to mitigate adverse effects, and it is not expected to impair antitumor activity. 


\section{Methods}

Bispecific antibodies. Bispecific antibodies were produced as described earlier $(4,39)$. Briefly, the "knob" and "hole" half antibodies were expressed in CHO cells and purified independently by Protein A affinity chromatography. After annealing the knob and hole in vitro at a 1:1 ratio in the presence of reduced glutathione, the assembled bispecific antibody was purified by hydrophobic interaction chromatography. The purified antibodies were characterized by mass spectrometry, analytical size exclusion chromatography, and SDS-PAGE, showing less than $1 \%$ aggregates and less than $\%$ homodimers. Anti-CD3 antibodies were generated at Genentech. Anti-HER2 arms were based on trastuzumab (humanized 4D5; ref. 40). HER2-TDB 3 included light chain N30S and heavy chain D98S substitutions in the hu4D5. All TDBs include N297G substitution in Fc.

HER2 binding. Recombinant human (Genentech) and cynomolgus monkey (Sino Biologicals) HER2 extracellular domain (ECD) were used to determine the binding affinity of anti-HER2/CD3 TDB for HER2 ECD by surface plasmon resonance (SPR) technology using a Biacore T200 instrument (GE Healthcare). Mouse anti-huIgG Fc (GE Healthcare) was covalently mobilized to all 4 flow cells (FCs) of a Series S Sensor Chip CM5 (GE Healthcare) using a standard amine coupling method. Anti-HER2/CD3 TDB was injected into FC2 and FC4, and the other 2 FCs - FC1 and FC3 - were used as in-line references for FC2 and FC4, respectively. Various concentrations of recombinant HER2 ECD were then injected into all 4 FCs for 2 minutes, and dissociation was allowed to proceed for 15 minutes. At the end of each association/dissociation cycle, the chip was regenerated using $3 \mathrm{M} \mathrm{MgCl}_{2}$. All experiments were conducted at $37^{\circ} \mathrm{C}$. Affinity $\left(\mathrm{K}_{\mathrm{D}}\right)$, dissociation rate constant $(\mathrm{kd})$, and association rate constant $(\mathrm{ka})$ were calculated from a 1:1 analyte binding model using Biacore Evaluation Software (GE Healthcare).

CD3 binding. Human and cynomolgus monkey CD3e peptides (Genentech) were used to determine the binding affinity of anti-HER2/CD3 TDBs for CD3 $\varepsilon$ by SPR using a Biacore T200 instrument. Biotinylated peptides were immobilized to FC2, FC3, and FC4 of a Series S Sensor Chip Streptavidin (GE Healthcare). No peptide was immobilized to FC1, which was used as an in-line reference for FC2, FC3, and FC4. Various concentrations of anti-HER2/CD3 were then injected into all FCs for 5 minutes, and dissociation was allowed to proceed for 2 minutes. At the end of each association/dissociation cycle, the chip was regenerated using $3 \mathrm{M} \mathrm{MgCl}_{2}$. All experiments were conducted at $37^{\circ} \mathrm{C}$. The off-rate for the low-affinity CD3 molecule was too fast to obtain kinetic information. Therefore, steady-state binding analysis was used to derive CD3 $\mathrm{K}_{\mathrm{D}}$ for all anti-HER2/CD3 molecules using Biacore Evaluation Software.

Cancer cell lines. Cell lines were authenticated/quality controlled using short tandem repeat (STR) profiling (Promega PowerPlex 16 System) and by high-throughput SNP profiling (Fluidigm multiplexed assays)

Isolation of human $C D 8^{+} T$ cells. Human PBMCs were separated from the blood of healthy volunteers using lymphocyte separation medium (MP biomedicals). CD8 ${ }^{+} \mathrm{T}$ cells were extracted from PBMC using human $\mathrm{CD}^{+}$Isolation Kit from Miltenyi Biotec (catalog 130-094-156) by negative selection.

$T D B$ binding to human $C D 8^{+} T$ cells. Ten thousand human $C D 8^{+} \mathrm{T}$ cells per well were placed in 96 well V-bottom plate (Corning) and incubated with anti-HER2/CD3 TDB as indicated on ice for 15 minutes. Cells were washed with FACS buffer (PBS, 2\% FBS, 1 mM EDTA) and stained with goat anti-human-IgG-APC (Jackson ImmunoResearch) and anti-CD8-FITC (BD Bioscience, clone HIT8a, catalog 555634) on ice for 15 minutes. Cells were washed twice with FACS buffer and propidium iodide (PI) was added to the final cell suspension. The binding activity was analyzed by flow cytometry, and the geometric means fluorescence of APC on cells in $\mathrm{CD}^{+}$gate was plotted.

Western blot analysis. To evaluate TCR signaling pathway activation, SKBR3 cells were incubated with splenic $\mathrm{CD}^{+} \mathrm{T}$ cells harvested from huCD3 TG mice at an effector/target cell (E:T) ratio of 3:1. Cells were treated with $1 \mu \mathrm{g} / \mathrm{mL}$ TDBs for indicated times. Phos-SLP-76 (Cell Signaling Technologies, catalog 13177), CD3 (Cell Signaling Techologies, catalog 85061), and HER2 (Dako, catalog A0485) antibodies were used for detection.

$T$ cell activation. All antibodies for flow cytometry cell staining were from BD Biosciences. Cocultures of human $\mathrm{CD}^{+} \mathrm{T}$ cells and SKBR3 cells (3:1 ratio) were incubated with the anti-HER2/CD3 TDBs for 24 hours and stained with anti-CD8-FITC (BD Bioscience, clone HIT8a, catalog 555634), anti-CD69-PE (BD Bioscience, clone FN50, catalog 555531), and anti-CD25-APC (BD Bioscience, clone M-A251, catalog 555434). CD69 and CD25 surface expression was detected on CD8 ${ }^{+} \mathrm{T}$ cells by flow cytometry. T cell activation was reported as percentage of $\mathrm{CD} 8^{+} \mathrm{T}$ cells that were $\mathrm{CD} 69^{+} \mathrm{CD} 25^{+}$.

Flow cytometry analysis of in vitro cell killing. Target cells were labeled with carboxyfluorescein succin- 
imidyl ester (CFSE; Thermo Fisher Scientific) following the manufacturer's instructions. Human CD8 ${ }^{+}$ $\mathrm{T}$ cells and CFSE-labeled target cells (3:1 ratio) were cocultured with the anti-HER2/CD3 TDBs for 24 hours. After treatment, cells were lifted with $0.25 \%$ trypsin/EDTA (Thermo Fisher Scientific) at $37^{\circ} \mathrm{C}$ for 5 minutes and washed once with FACS buffer. PI was added to the final cell suspension. The number of live target cells was counted by flow cytometry using a gate of $\mathrm{CFSE}^{+} \mathrm{PI}^{-}$. Killing activity was calculated as: ([number of live target cells without treatment - number of live target cells with treatment]/[number of live target cells without treatment]) $\times 100 \%$.

In vitro killing of human and cynomolgus monkey HER2-expressing $\mathrm{CHO}$ cells. $\mathrm{CHO}$ cells were transfected with full-length human or cynomolgus monkey HER2 using Lipofectamine 2000 (Thermo Fisher Scientific). Twenty-four hours after transfection, cells were stained with APC mouse anti-human HER-2/neu (clone NEU 24.7, catalog 340554, BD Biosciences), and HER2 ${ }^{+}$cells were sorted by FACS. Five thousand HER2 ${ }^{+} \mathrm{CHO}$ cells were incubated at $37^{\circ} \mathrm{C}$ overnight before adding 15,000 human $\mathrm{CD}^{+}$cells and treating with the HER2/CD3 TDBs for 48 hours. Viability was assayed using Cell Titer-Glo (Promega).

In vitro cytokine release. RBCs were lysed from heparin-treated healthy donor blood using ammonium chloride potassium (ACK) red cell lysis buffer. Four-hundred thousand whole blood cells were incubated with MCF7 cells and the HER2-TDBs for 20 hours in 10:1 E:T ratio. Cytokine release from supernatants was analyzed by Bio-Plex Pro Human Cytokine Assays (Bio-Rad).

In vivo efficacy in treatment of KPL4 tumors. Three million KPL4 cells were inoculated into the thoracic mammary fat pad of 8-week-old NSG mice (The Jackson Laboratory; stock no. 005557) in HBSS/matrigel in a volume of $0.2 \mathrm{~mL}$. One day later, $10 \times 10^{6}$ human PBMCs cultured overnight in nonactivating conditions were injected i.p. The tumor-bearing mice were randomized into control and treatment groups based on the tumor volumes $\left(\sim 200 \mathrm{~mm}^{3}\right)$, and dosing was initiated at day 0 . All treatments were administered i.v. by tail vein injection. Antibodies were diluted in Histidine Buffer \#8 (vehicle; Histidine Acetate $20 \mathrm{mM}$, Sucrose $240 \mathrm{mM}$, TW-20 0.02\%, pH 5.5).

In vivo efficacy in treatment of 2-tumor model. Mice were inoculated with $3 \times 10^{6} \mathrm{HER} 2$-amplified KPL4 cells suspended in $0.2 \mathrm{~mL}$ of HBSS/Matrigel $(1: 1, \mathrm{v} / \mathrm{v})$ in the left thoracic mammary fat pad and $5 \times 10^{6}$ low HER2-expressing HT55 cells suspended in $0.1 \mathrm{~mL}$ of HBSS in the right unilateral thoracic flank. Human PBMCs were inoculated as described above, and treatments were initiated 9 days after cell inoculation (day 0).

huHER2.huCD3 transgenic mice. Mice expressing human WT HER2 in mammary epithelial cells and human CD3e in T cells have been previously described (5).

Mouse tumor tissue preparation for flow cytometry analysis. Processing of MMTVhuHER2 tumors for flow cytometry was done using the Human Tumor Dissociation Kit (Miltenyi Biotec) according to manufacturer's instruction. Briefly, tumors were cut into small pieces of 2-4 $\mathrm{mm}$ and transferred to gentleMACS C-tubes (Miltenyi Biotec) in $5 \mathrm{~mL}$ of fresh-made ice-cold RPMI medium with enzyme mix. C-tubes were placed onto gentleMACS Octo Dissociator with Heaters. GentleMACS program 37C_m_TDK_2 was used to dissociate tumors. Samples were filtered through a $70-\mu \mathrm{m}$ cell strainer and spun down at $200 \mathrm{x} g$ for 5 minutes. Single-cell suspensions were prepared in FACS buffer and stained against the indicated markers for flow cytometric analysis. The live/dead fixable near-infrared dye (Invitrogen) was used to exclude dead cells. For intracellular staining of Ki67, cells were first stained with cell surface markers and then fixed and permeabilized with fixation and permeabilization buffers (eBioscience) before stained with anti-Ki67 (BD Bioscience, clone B56, catalog 561277).

Mouse cytokine analysis. MMTV.huHER2.FVB/n transgenic female mice were treated with HER2TDBs at $0.5 \mathrm{mg} / \mathrm{kg}$. Mouse blood was collected in serum separator tubes (BD Biosciences) and centrifuged at $1,200 \mathrm{x} g$ for 10 minutes. The sera were stored at $-80^{\circ} \mathrm{C}$. Cytokines in serum were analyzed using the mouse cytokine Luminex assay (Bio-Rad) according to the manufacturer instructions.

Cynomolgus monkey tolerability studies. The toxicology studies were conducted at Charles River Laboratories (CRL); all animals were experimentally naive, purpose-bred cynomolgus monkeys (Macaca fascicularis). A single-dose tolerability study in female cynomolgus monkeys was performed comparing HER2TDB $1(n=2)$ and HER2-TDB $2(n=1)$. Both molecules were administered via i.v. slow bolus infusion at a dose level of $0.5 \mathrm{mg} / \mathrm{kg}$. A second multiple dose tolerability study was conducted in female cynomolgus monkeys comparing HER2-TDB 1 and HER2-TDB 3. Both molecules were administered via i.v. infusion ( 1 hour) at a dose level of $0.5 \mathrm{mg} / \mathrm{kg}$ or $1.5 \mathrm{mg} / \mathrm{kg}(n=4)$ administered on days 0 and 7 , with reversibility assessment on day 35. A third, dose fractionation study was performed in female cynomolgus monkeys 
with HER2-TDB 3 as a fractionated dose to achieve a similar exposure as the previous multidose study. Three animals were dosed with $0.1(n=2)$ or $0.2 \mathrm{mg} / \mathrm{kg}(n=1)$ on day $0,0.2 \mathrm{mg} / \mathrm{kg}$ on day 1 , and $0.4 \mathrm{mg} /$ $\mathrm{kg}$ on day 7 via i.v. infusion (1 hour).

In-life evaluations in all studies included clinical signs, body weights, food consumption, respiratory rate, body temperature, clinical pathology (e.g., hematology, coagulation, and serum chemistry), immunology parameters (immunophenotyping and cytokines), toxicokinetics, gross necropsy findings, organ weights, and histopathology.

Serum test article concentrations at multiple time points were determined by enzyme-linked immunosorbent assay, and concentration-time profiles were used to estimate pharmacokinetic parameters using WinNonlin software (Pharsight). Cytokine assessment was conducted by Luminex on plasma samples and included IL-1RA, IL-2, IL-6, IFN- $\gamma$, TNF- $\alpha$, and MCP-1. Immunophenotyping by flow cytometry was used to quantify $\mathrm{T}$ cell populations and activation markers (CD69) in the peripheral blood. Immunophenotyping and cytokine quantitation are further described in the Supplemental Methods.

Statistics. Welch's $t$ test (unpaired with parametric and unequal variances $t$ test) was used to test for differences between groups. Results were determined to be significant at $P<0.05$. Statistical analyses were performed using GraphPad Prism version 7.

Study approval. All in vivo mouse experimental procedures conformed to the guiding principles of the American Physiology Society and were approved by Genentech's IACUC. All toxicology study procedures were conducted according to written study protocols and testing facility standard operating procedures, approved by the CRL IACUC, and performed in compliance with the Animal Welfare Act, the Guide for the Care and Use of Laboratory Animals (National Academies Press, 2011), and the Office of Laboratory Animal Welfare.

\section{Author contributions}

KS, CLZdZ, LKS, VC, FZ, MH, RC, JL, MM, XC, JJ, JL, RY, DS, JY, MO, NOD, KT, MRJ, DE, GL, MSD, RP, and TTJ contributed to the study design, data analysis, and interpretation. . MH, RC, JL, MM, XC, JJ, JL, RY, and DS performed the experiments. KS, CLZdZ, LKS, RP, and TTJ wrote the manuscript. Project management was contributed by GL.

Address correspondence to: Teemu T. Junttila or Rodney Prell, Genentech Inc. 1 DNA Way, South San Francisco, California 94080, USA. Phone: 650.225.1000; Email: junttila.teemu@gene.com (T.T. Junttila); rodneyp@gene.com (R. Prell).

CLZdZ's present address is: Amgen Inc., South San Francisco, California, USA.

MM's present address is: Distributed Bio, South San Francisco, California, USA.

XC's present address is: Virtuoso Therapeutics, San Mateo, California, USA.

MSD's present address is: Denali, South San Francisco, California, USA.

1. Baselga J, Coleman RE, Cortés J, Janni W. Advances in the management of HER2-positive early breast cancer. Crit Rev Oncol Hematol. 2017;119:113-122.

2. Slamon DJ, Clark GM, Wong SG, Levin WJ, Ullrich A, McGuire WL. Human breast cancer: correlation of relapse and survival with amplification of the HER-2/neu oncogene. Science. 1987;235(4785):177-182.

3. Connell CM, Doherty GJ. Activating HER2 mutations as emerging targets in multiple solid cancers. ESMO Open. 2017;2(5):e000279.

4. Junttila TT, et al. Antitumor efficacy of a bispecific antibody that targets HER2 and activates T cells. Cancer Res. 2014;74(19):5561-5571.

5. Li J, et al. IFN $\gamma$-induced Chemokines Are Required for CXCR3-mediated T-Cell Recruitment and Antitumor Efficacy of AntiHER2/CD3 Bispecific Antibody. Clin Cancer Res. 2018;24(24):6447-6458.

6. Bargou R, et al. Tumor regression in cancer patients by very low doses of a T cell-engaging antibody. Science. 2008;321(5891):974-977.

7. Brentjens RJ, et al. CD19-targeted T cells rapidly induce molecular remissions in adults with chemotherapy-refractory acute lymphoblastic leukemia. Sci Transl Med. 2013;5(177):177ra38.

8. Kalos M, et al. T cells with chimeric antigen receptors have potent antitumor effects and can establish memory in patients with advanced leukemia. Sci Transl Med. 2011;3(95):95ra73.

9. Kantarjian H, et al. Blinatumomab versus Chemotherapy for Advanced Acute Lymphoblastic Leukemia. N Engl J Med. 2017;376(9):836-847.

10. Maude SL, et al. Chimeric antigen receptor T cells for sustained remissions in leukemia. N Engl J Med. 2014;371(16):1507-1517. 
11. Turtle CJ, et al. Immunotherapy of non-Hodgkin's lymphoma with a defined ratio of CD8+ and CD4+ CD19-specific chimeric antigen receptor-modified T cells. Sci Transl Med. 2016;8(355):355ra116.

12. Fan F, Zhao W, Liu J, He A, Chen Y, Cao X. Durable remissions with BCMA-specific chimeric antigen receptor (CAR)-modified T cells in patients with refractory/relapsed multiple myeloma. J Clin Oncol. 2017;35(18_suppl):LBA3001.

13. Fiedler WM, et al. A phase I study of EpCAM/CD3-bispecific antibody (MT110) in patients with advanced solid tumors. $J$ Clin Oncol. 2012;30(15_suppl):2504.

14. Haense $\mathrm{N}$, et al. A phase I trial of the trifunctional anti Her $2 \times$ anti CD3 antibody ertumaxomab in patients with advanced solid tumors. BMC Cancer. 2016;16:420.

15. Johnson LA, et al. Gene therapy with human and mouse T-cell receptors mediates cancer regression and targets normal tissues expressing cognate antigen. Blood. 2009;114(3):535-546.

16. Lutterbuese R, et al. T cell-engaging BiTE antibodies specific for EGFR potently eliminate KRAS- and BRAF-mutated colorectal cancer cells. Proc Natl Acad Sci USA. 2010;107(28):12605-12610.

17. Morgan RA, Yang JC, Kitano M, Dudley ME, Laurencot CM, Rosenberg SA. Case report of a serious adverse event following the administration of T cells transduced with a chimeric antigen receptor recognizing ERBB2. Mol Ther. 2010;18(4):843-851.

18. Parkhurst MR, et al. T cells targeting carcinoembryonic antigen can mediate regression of metastatic colorectal cancer but induce severe transient colitis. Mol Ther. 2011;19(3):620-626.

19. Press MF, Cordon-Cardo C, Slamon DJ. Expression of the HER-2/neu proto-oncogene in normal human adult and fetal tissues. Oncogene. 1990;5(7):953-962.

20. Slaga D, et al. Avidity-based binding to HER2 results in selective killing of HER2-overexpressing cells by anti-HER2/CD3. Sci Transl Med. 2018;10(463):eaat5775.

21. Leong SR, et al. An anti-CD3/anti-CLL-1 bispecific antibody for the treatment of acute myeloid leukemia. Blood. 2017;129(5):609-618.

22. Sharma SK, et al. Fc-Mediated Anomalous Biodistribution of Therapeutic Antibodies in Immunodeficient Mouse Models. Cancer Res. 2018;78(7):1820-1832.

23. Shultz LD, et al. Human lymphoid and myeloid cell development in NOD/LtSz-scid IL2R gamma null mice engrafted with mobilized human hemopoietic stem cells. J Immunol. 2005;174(10):6477-6489.

24. de la Hera A, Müller U, Olsson C, Isaaz S, Tunnacliffe A. Structure of the T cell antigen receptor (TCR): two CD3 epsilon subunits in a functional TCR/CD3 complex. J Exp Med. 1991;173(1):7-17.

25. Finkle D, et al. HER2-targeted therapy reduces incidence and progression of midlife mammary tumors in female murine mammary tumor virus huHER2-transgenic mice. Clin Cancer Res. 2004;10(7):2499-2511.

26. Gust J, Taraseviciute A, Turtle CJ. Neurotoxicity Associated with CD19-Targeted CAR-T Cell Therapies. CNS Drugs. 2018;32(12):1091-1101.

27. Saber H, Del Valle P, Ricks TK, Leighton JK. An FDA oncology analysis of CD3 bispecific constructs and first-in-human dose selection. Regul Toxicol Pharmacol. 2017;90:144-152.

28. Florido R, Smith KL, Cuomo KK, Russell SD. Cardiotoxicity From Human Epidermal Growth Factor Receptor-2 (HER2) Targeted Therapies. J Am Heart Assoc. 2017;6(9):e006915.

29. Sato J, Doi T, Kanno T, Wako Y, Tsuchitani M, Narama I. Histopathology of incidental findings in cynomolgus monkeys ( macaca fascicularis ) used in toxicity studies. J Toxicol Pathol. 2012;25(1):63-101.

30. Negro A, Brar BK, Lee KF. Essential roles of Her2/erbB2 in cardiac development and function. Recent Prog Horm Res. 2004;59:1-12

31. Klein PM, Dybdal N. Trastuzumab and cardiac dysfunction: update on preclinical studies. Semin Oncol. 2003;30(5 Suppl 16):49-53.

32. Li J, et al. Membrane-Proximal Epitope Facilitates Efficient T Cell Synapse Formation by Anti-FcRH5/CD3 and Is a Requirement for Myeloma Cell Killing. Cancer Cell. 2017;31(3):383-395.

33. Mandikian D, et al. Relative Target Affinities of T-Cell-Dependent Bispecific Antibodies Determine Biodistribution in a Solid Tumor Mouse Model. Mol Cancer Ther. 2018;17(4):776-785.

34. Irvine DJ, Purbhoo MA, Krogsgaard M, Davis MM. Direct observation of ligand recognition by T cells. Nature. 2002;419(6909):845-849.

35. Purbhoo MA, Irvine DJ, Huppa JB, Davis MM. T cell killing does not require the formation of a stable mature immunological synapse. Nat Immunol. 2004;5(5):524-530.

36. Sykulev Y, Joo M, Vturina I, Tsomides TJ, Eisen HN. Evidence that a single peptide-MHC complex on a target cell can elicit a cytolytic T cell response. Immunity. 1996;4(6):565-571.

37. Zuch de Zafra CL, et al. Targeting Multiple Myeloma with AMG 424, a Novel Anti-CD38/CD3 Bispecific T-cell-recruiting Antibody Optimized for Cytotoxicity and Cytokine Release. Clin Cancer Res. 2019;25(13):3921-3933.

38. Yuraszeck T, Kasichayanula S, Benjamin JE. Translation and Clinical Development of Bispecific T-cell Engaging Antibodies for Cancer Treatment. Clin Pharmacol Ther. 2017;101(5):634-645.

39. Sun LL, et al. Anti-CD20/CD3 T cell-dependent bispecific antibody for the treatment of B cell malignancies. Sci Transl Med. 2015;7(287):287ra70.

40. Carter P, et al. Humanization of an anti-p185HER2 antibody for human cancer therapy. Proc Natl Acad Sci USA 1992;89(10):4285-4289. 